\title{
Laparoscopic Repair of Incarcerated Intersigmoid Hernia
}

\author{
Aideen Campbell, MBBS, and Gary K. Atkin, MD \\ Department of Surgery, Barnet and Chase Farm Hospitals NHS Trust, Barnet, UK (all authors).
}

\begin{abstract}
Introduction: An intersigmoid hernia is a type of internal hernia and is a rare cause of small-bowel obstruction. It occurs because of a congenital peritoneal defect at the root of the sigmoid mesocolon called the intersigmoid fossa.

Case Description: A case of intersigmoid hernia causing acute small-bowel obstruction is presented and a technique for the laparoscopic management of an incarcerated intersigmoid hernia is described.

Discussion: This is the first reported case of an intersigmoid hernia being repaired by a totally laparoscopic approach and highlights the utility of laparoscopy in acute small-bowel obstruction, particularly when the patient has not had previous abdominal surgery.
\end{abstract}

Key Words: Intestinal obstruction, Intersigmoid hernia, Laparoscopy.

Citation Campbell A, Atkin GK. Laparoscopic repair of incarcerated intersigmoid hernia. CRSLS e2013.00214. DOI: 10.4293/CRSLS.2013.00214.

Copyright (C) 2015 by SLS, Society of Laparoendoscopic Surgeons. This is an open-access article distributed under the terms of the Creative Commons Attribution-Noncommercial-ShareAlike 3.0 Unported license, which permits unrestricted noncommercial use, distribution, and reproduction in any medium, provided the original author and source are credited.

Address correspondence to: Gary K. Atkin, MD, Department of Surgery, Barnet and Chase Farm Hospitals NHS Trust, Wellhouse Lane, Barnet, UK EN5 5HB. Telephone: (+44) 208-216-5453, Fax: (+44) 208-216-5447, E-mail: gary.atkin@nhs.net

\section{INTRODUCTION}

Internal hernias are an uncommon, but important, cause of small-bowel obstruction. An intersigmoid hernia is a rare type of internal hernia and occurs at the intersigmoid fossa. This is a congenital defect in the attachment between the sigmoid mesocolon and the peritoneum of the paracolic gutter. Making a diagnosis of intersigmoid hernia causing small-bowel obstruction can be difficult and is usually made during laparotomy. ${ }^{1}$ This is the first reported case of the complete laparoscopic reduction and repair of an incarcerated intersigmoid hernia, and a method of management is described along with a discussion on the role of laparoscopy in acute small-bowel obstruction.

\section{Case Description}

A 56-year-old woman presented emergently with a 4-day history of generalized colicky abdominal pain and bilious vomiting. She had no significant past medical history and had not had any previous abdominal surgery. Clinical examination revealed a distended, soft abdomen with mild central tenderness and no signs of peritonism. There were no external hernias evident. Initial blood test results were all within normal ranges. A plain abdominal radiograph demonstrated multiple dilated loops of small bowel, and an erect chest radiograph was unremarkable. To clarify the etiology and level of obstruction, the patient underwent a computed tomography (CT) scan of the abdomen and pelvis with intravenous contrast. This revealed a dilated proximal small bowel with a transition point in the left iliac fossa, without any visible mass. The provisional diagnosis was of a congenital adhesional band causing small-bowel obstruction.

Diagnostic laparoscopy was considered safe to perform because of an acceptable level of small-bowel distention on clinical and radiologic assessment, and this occurred on the day of admission. An open entry method was used and an 11-mm port was placed at the umbilicus. Two further 5-mm ports were placed under direct vision in the suprapubic and right iliac fossa regions. Laparoscopy revealed complete small-bowel obstruction caused by an internal hernia between the root of the sigmoid mesocolon and the peritoneum overlying the left pelvic brim (Figure 1). The hernial defect was $3 \mathrm{~cm}$ in diameter, and the sac was approximately $6 \mathrm{~cm}$ in length, long enough to accommodate a full-thickness loop of small intestine. The 


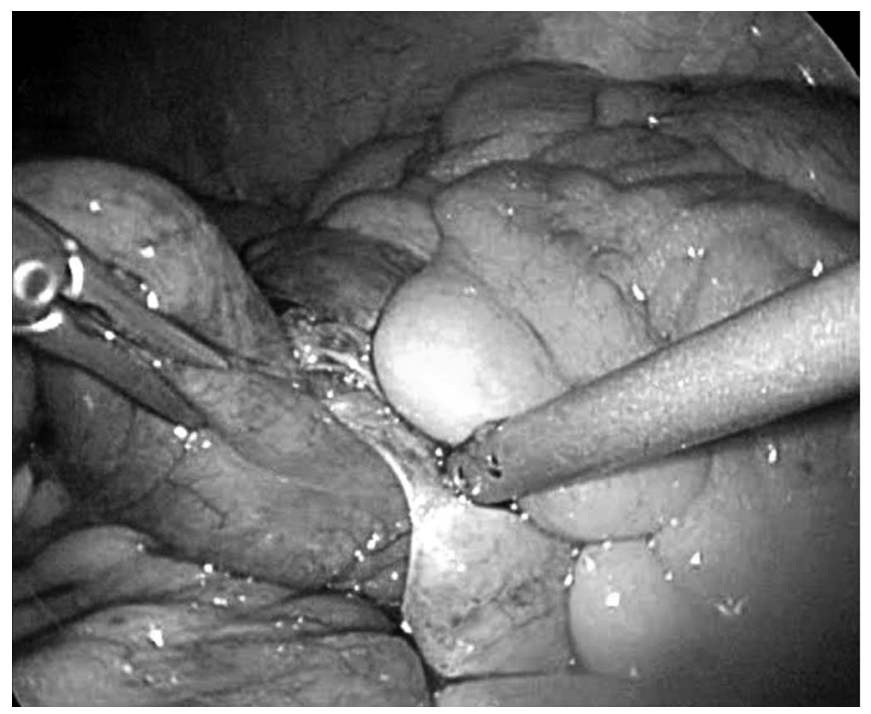

Figure 1. An intraoperative view of the internal hernia at the root of the sigmoid mesocolon containing incarcerated loop of the small intestine.

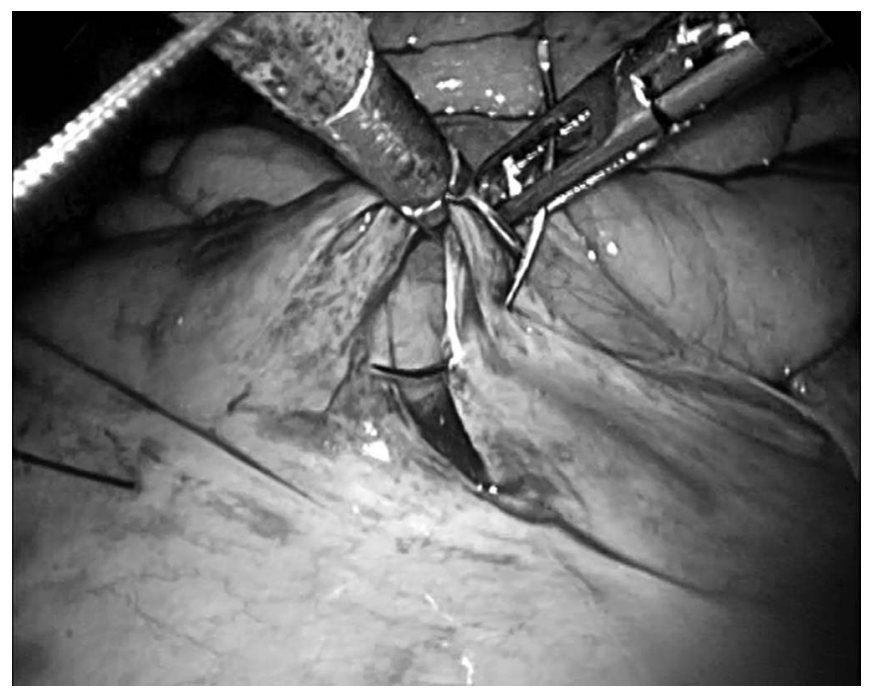

Figure 2. An intraoperative view showing intracorporeal suture repair of the hernial defect.

bowel was reduced by gentle traction and was viable; thus, resection was unnecessary. The hernial defect was closed with intracorporeal suturing using 2-0 polyglactin 910 sutures (Vicryl, Ethicon, Livingston, UK) (Figure 2). The duration of the procedure was 60 minutes.

The patient returned to the ward and required minimal analgesia postoperatively. Her nasogastric tube and urinary catheter were removed the following day, and she was fit for discharge 36 hours after her surgery. She was followed up for 6 months and no further problems developed.

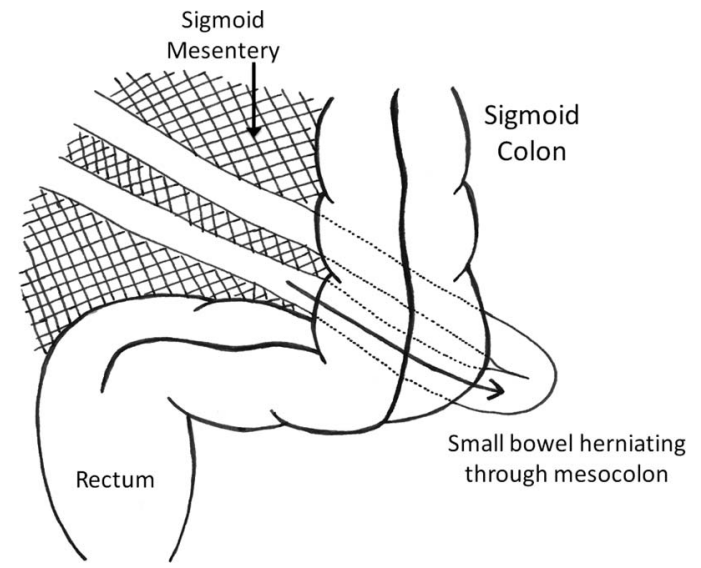

Figure 3. Transmesosigmoid hernia showing the small bowel herniating through the defect in the medial and lateral sigmoid peritoneal folds.

\section{DISCUSSION}

The intersigmoid fossa is a peritoneal defect at the root of the sigmoid mesocolon, typically located at a point overlying the left ureter. ${ }^{2}$ It may occur as a depression or as a full peritoneal defect, ${ }^{3}$ and it represents a localized failure of fusion along Toldt line between the visceral sigmoid peritoneum and the parietal peritoneum of the posterior abdominal wall. ${ }^{4}$ Its prevalence at autopsy has been reported to be $65 \% 0^{5}$ and so may be noted incidentally during abdominal surgery.

Pathologic sigmoid hernia was first described in $1885,{ }^{6}$ and since then, it has been reported infrequently. It is a form of internal hernia occurring within or adjacent to the sigmoid mesocolon. These sigmoid mesocolon hernias were divided by Benson and Killen into 3 subtypes-intersigmoid, transmesosigmoid, and intramesosigmoid. ${ }^{4}$ Transmesosigmoid hernias occur as the result of a defect through the medial and lateral visceral peritoneum overlying the sigmoid mesocolon (Figure 3). An intramesosigmoid hernia occurs because of a defect in the lateral peritoneal fold only, with bowel herniating into the sigmoid mesocolon (Figure 4). Although historically an intersigmoid hernia was thought to be most common subtype, ${ }^{4}$ a recent literature review suggests that intramesosigmoid hernia is the most common. ${ }^{1}$

Internal hernias are the cause of small intestinal obstruction in less than $4 \%$ of cases. ${ }^{2}$ The incidence is much higher, however, after gastric bypass surgery. ${ }^{7}$ Sigmoid mesocolon hernias comprise only $5 \%$ of internal hernias and are a rare cause of small-bowel obstruction. ${ }^{2}$ They are difficult to diagnose on clinical grounds, but suggestive CT findings include a U- or C-shaped cluster of small bowel 


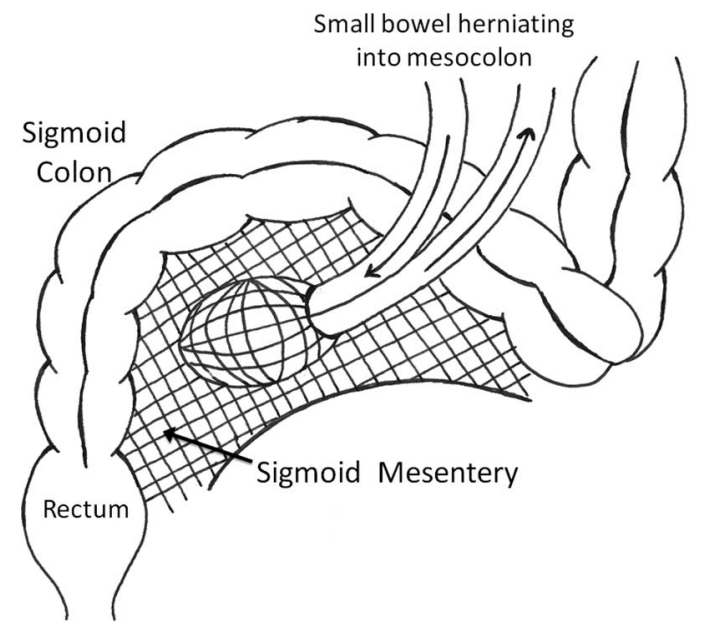

Figure 4. Intramesosigmoid hernia showing the small bowel incarcerated within the sigmoid mesentery after herniating through the defect in the lateral sigmoid peritoneal fold.

posterior and lateral to the sigmoid colon. 5 There are no definitive features to differentiate the subtype of sigmoid mesocolon hernia on imaging. ${ }^{8}$

There is an increasing interest in the laparoscopic management of acute small-bowel obstruction. For adhesional obstruction, meta-analysis of retrospective, nonrandomized data has shown a reduced rate of complications and postoperative ileus compared with an open approach. ${ }^{9}$ The rate of conversion, however, is up to $50 \% .{ }^{10}$ There is little evidence to guide management of nonadhesional small-bowel obstruction, and so the role of laparoscopy is not yet defined. Traditional surgical teaching states that these patients usually require surgical intervention. Indeed, previous attempts at nonoperative management of incarcerated intersigmoid hernias have been unsuccessful., ${ }^{1,2}$ In these previously reported cases, laparotomy was eventually required and the length of stay was 8 days, compared with 36 hours after the laparoscopic repair in the present case.

Treatment of transmesosigmoid and intramesosigmoid hernias by laparoscopy has been reported before, ${ }^{11-13}$ but this is the first reported case of an intersigmoid hernia being completely managed laparoscopically. With sufficient expertise, laparoscopy is the ideal approach when the etiology of acute small-bowel obstruction is uncertain, providing the degree of bowel distention is acceptable and there is no evidence of bowel perforation. In acute small-bowel obstruction of varying etiology, laparoscopy has been shown to have a diagnostic accuracy of $97 \%$, with $72 \%$ of patients being treated with laparoscopy or a small targeted incision, and an acceptable conversion rate of $28 \% .{ }^{14}$ Laparoscopy also has the additional benefit of reducing the risk of subsequent symptomatic adhesion formation. ${ }^{15}$

\section{References:}

1. Nihon-Yanagi Y, Ooshiro M, Osamura A, et al. Intersigmoid hernia: report of a case. Surg Today. 2010;40(2):171-175.

2. Harrison OJ, Sharma RD, Niayesh MH. Early intervention in intersigmoid hernia may prevent bowel resection-A case report. Int J Surg Case Rep. 2011;2(8):282-284.

3. Takeyama N, Gokan T, Ohgiya Y, et al. CT of internal hernias. Radiographics. 2005;25(4):997-1015.

4. Benson JR, Killen DA. Internal hernias involving the sigmoid mesocolon. Ann Surg. 1964;159:382-384.

5. Martin LC, Merkle EM, Thompson WM. Review of internal hernias: radiographic and clinical findings. AJR Am J Roentgenol. 2006;186(3):703-717.

6. Vickers DM, Fortuine ST. Intersigmoid hernia. Ann Surg. 1933;97(5):713-716.

7. Hamdan K, Somers S, Chand M. Management of late postoperative complications of bariatric surgery. Br J Surg. 2011; 98(10):1345-1355.

8. Mathieu D, Luciani A. Internal abdominal herniations. $A J R$ Am J Roentgenol. 2004;183(2):397-404.

9. Li MZ, Lian L, Xiao LB, et al. Laparoscopic versus open adhesiolysis in patients with adhesive small-bowel obstruction: a systematic review and meta-analysis. Am J Surg. 2012;204(5):779-786.

10. Agresta F, Ansaloni L, Baiocchi GL, et al. Laparoscopic approach to acute abdomen from the Consensus Development Conference of the Societa Italiana di Chirurgia Endoscopica e nuove tecnologie (SICE), Associazione Chirurghi Ospedalieri Italiani (ACOI), Societa Italiana di Chirurgia (SIC), Societa Italiana di Chirurgia d'Urgenza e del Trauma (SICUT), Societa Italiana di Chirurgia nell'Ospedalita Privata (SICOP), and the European Association for Endoscopic Surgery (EAES). Surg Endosc. 2012;26(8):2134-2164.

11. Jimmy J, Wani SV, Shetty VV, et al. Laparoscopic management of small bowel obstruction caused by a sigmoid mesocolic hernia. J Minim Access Surg. 2011;7(4):236-238.

12. Van der Mieren G, de Gheldere C, Vanclooster P. Transmesosigmoid hernia: report of a case and review of the literature. Acta Chir Belg. 2005;105(6):653-655.

13. Puri V, Bertellotti RP, Garg N, et al. Intramesosigmoid hernia: a rare type of congenital internal hernia. Hernia. 2007;11(5):463-465.

14. Kirshtein B, Roy-Shapira A, Lantsberg L, et al. Laparoscopic management of acute small bowel obstruction. Surg Endosc. 2005;19(4):464-467.

15. Burns EM, Currie A, Bottle A, et al. Minimal-access colorectal surgery is associated with fewer adhesion-related admissions than open surgery. Br J Surg. 2013;100(1):152-159. 